\title{
Analysis for the bargaining confidence degree in relocation disputes based on the Rubinstein bargaining model
}

\author{
Zhongfu Qin ${ }^{\text {a }}$, Mingtao Zhang ${ }^{\mathrm{b}}$, Xiaolong Lei ${ }^{\mathrm{c}}$ \\ College of Civil Engineering and Architecture Zhejiang University, Hangzhou, China \\ aquinzhongfu@zju.edu.cn, bjalioncheung@163.com, 'Ixl5358949@126.com
}

Keywords: Relocation dispute, Bargaining confidence degree, Rubinstein bargaining model, Information asymmetry and dynamics.

\begin{abstract}
The relocation dispute is a common type of dispute issues. The core of the relocation dispute usually lies in the divergence on the amount of the relocation compensation and the process of fighting for it among all the parties. Aiming at the limitations of the Rubinstein bargaining model for the analysis of the bargaining in relocation disputes, this paper, based upon the information asymmetry and dynamics of the relocation issue, redefines the discount factor as the bargaining confidence degree. In the meanwhile, this paper analyzes the interaction and restriction relationship between the bargaining confidence degree and the subjective factors of the bargaining player under the condition of information asymmetry and dynamics.
\end{abstract}

\section{Introduction}

In recent years, the cases incurred by the conflict of interests, the intensification of contradictions and malignant events which are triggered by the city housing relocation have been seen a lot. "The most conspicuous nail house in history" in Chongqing Yangjiaping and "the most expensive nail household Cai Zhuxiang case" in Shenzhen named by domestic media all explain that the proper mediation of housing relocation disputes is an integral factor in securing and promoting the development of the relocation work.

When the research on the dispute of the relocation has become the limelight in recent years, many research breakthroughs have been made based upon the game theory as the main means. X. B. Peng ${ }^{[1]}$ and $\mathrm{Z}$. S. $\mathrm{Li}^{[2]}$ respectively point out the importance of the analysis about the role orientation, strategy formulation and adjustment and the interplay of interests by means of the basic idea of the game theory. Concerning the aspects of analysis method of game theory, W. Zhong ${ }^{[3]}$, analyses the interest influence on parties of a dispute brought by anti-levy, bribery, petition by means of prisoner's dilemma model for relocation, thus putting forward reasonable suggestions for solving conflicts in land acquisition.

In relocation disputes, the focus of the parties during the dispute is often on the amount of the relocation compensation and the process of striving for compensation amount can be regarded as a bargaining. As a classical analysis method, the Rubinstein bargaining model has been widely used in many fields. Bargaining game concept was first proposed by N. John ${ }^{[4]}$. Subsequently, A. Rubinstein ${ }^{[5]}$ established the Rubinstein bargaining model under the condition of complete information alternate bid. But the process of the bargaining process is a dynamic process. In the bargaining process, the players may be affected by such factors as reserve price, time pressure, the opponent type, rivals concessions mode and the extent of concessions, expected future rival bids and at the same time will also be influenced by subjective moods like reciprocal psychology, fair psychology, and self-satisfaction of players ${ }^{[6]}$. Besides these subjective factors, the influence of information asymmetry phenomenon in realistic problems has been indicated in the relocation disputes, as well as many fields such as market for investment ${ }^{[7]}$ and trading dispute ${ }^{[8]}$. All of these problems indicate that it is necessary to figure out the main factor which affects the bargaining process in the relocation disputes based upon the influence of subjective factors and the information asymmetry and dynamics. 


\section{The limitation of the Rubinstein bargaining model in relocation disputes}

Introduction of the Rubinstein bargaining model. Rubinstein explored deep into the bargaining model, simulated the basic and indefinite complete bargaining process based on the problem of "dividing the cake" and offered the alternating bargaining model under the condition of complete information, namely the Rubinstein bargaining model. In this model, player 1 and player 2 segment the cake whose size is $\pi(\pi>0)$, and the allocation scheme is put forward by player 1 by turns and the agreement is made until one of them accept another's proposal (or final agreement cannot be reached when negotiations break down). Based upon the differences of the final acceptance scheme and the Rubinstein, introducing the conception of factors concerning the level of patience of parties, gives the most optimal bidding scheme when different participants bargain.

When player 1's offer is accepted by player 2 after odd round bargaining, player 1 should require the share of $\frac{1-\delta_{2}}{1-\delta_{1} \delta_{2}} \pi$, and player 2 should accept any offer which provides the share not less than $\frac{\delta_{1}\left(1-\delta_{2}\right)}{1-\delta_{1} \delta_{2}} \pi$. When player 2's offer is accepted by player 1 after even round bargaining, player 2 should require the share of $\frac{1-\delta_{1}}{1-\delta_{1} \delta_{2}} \pi$, and player 1 should accept any offer which provides the share not less than $\frac{\delta_{2}\left(1-\delta_{1}\right)}{1-\delta_{1} \delta_{2}} \pi . \delta_{1}$ And $\delta_{2}$ respectively represent the discount factor of player 1 and player 2 .

The limitation of the Rubinstein bargaining model in relocation disputes. Firstly, the process of the bargaining is dynamical. Two players (the demolition and the household) take turns to give the bargaining price until the agreement is reached or the negotiation breaks. So the players will adjust their own bargaining price dynamically.

Secondly, the bargaining processes under the condition of information asymmetry. The players can only know their own ability to bargain (discount factor) but only guess the discount factor of other player with some method. Sometimes one player will sent some false signal to lead other player to draw a wrong conclusion, so the player can benefit himself mostly.

Besides, the players cannot be rational. The discount factor can only represent the economic influence, but the attitude and the ability of the bargaining players will change when they feel happy or thankful, angry or scared and so on, which the definition of discount factor cannot help with.

\section{The analysis for the bargaining confidence degree in relocation disputes}

The definition and expression of the bargaining confidence degree in relocation disputes. Based on the analysis for the using of the Rubinstein bargaining model in the relocation disputes, we can know that the discount factor decides the final bargaining price. But we have to take the limitations of this model into consideration. So we will redefine the discount factor as the bargaining confidence degree in the relocation disputes, expressed as $\delta$. Similarly as the discount factor $\delta \in[0,1] \delta=0$ means that the player has completely lost the ability to negotiate, and the other player will decide the final bargaining price that both players agree. $\delta=1$ Means that the player is confident enough to make decision on his own bargaining price.

Due to the condition of information asymmetry, we use $\delta_{\mathrm{ii}}$ and $\delta_{\mathrm{ij}}$ to represent the confidence degree of i (demolition) and i's confidence degree estimated by j (household) respectively. And $\delta_{\mathrm{jj}}$ $\delta_{\mathrm{ji}}$ will be used to represent the confidence degree of $\mathrm{j}$ (household) andj's confidence degree estimated by $i$ (demolition) respectively. So the differences between $\delta_{\mathrm{ii}}$ and $\delta_{\mathrm{ij}}$, $\delta_{\mathrm{jj}}$ and $\delta_{\mathrm{ji}}$ will be caused by the information asymmetry

Due to the effect of the dynamic, we can use $\delta(t)$ to represent the confidence degree of the player in round $t$ of bargaining. So $\delta_{i i}(t)$ and $\delta_{i j}(t)$ to represent the confidence degree of $i$ (demolition) and $i$ 's confidence degree estimated by $\mathrm{j}$ (household) respectively in round $t$ of bargaining. And $\delta_{j j}(t)$ $\delta_{j i}(t)$ will be used to represent the confidence degree of $\mathrm{j}$ (household) andj's confidence degree estimated by $i$ (demolition) respectively in round $t$ of bargaining, and $t \geq 1$.

The calculation formula of the bargaining confidence degree in relocation disputes. Based on the discussion above, we know that it is necessary to take the influence of irrationality of the players 
in the relocation disputes into consideration. So we will give some new definitions before we calculate the bargaining confidence degree in relocation disputes.

The coefficient of the cooperative emotions $\alpha$, represent the degree of influence effecting on the players by the cooperative emotions. For the player $i$ in round $t$ of bargaining, when $\delta_{i i}(t-1)>$ $\delta_{j i}(t-1)$, player $i$ is more confident than $j$, so the player $i$ will feel happy, pity or just thankful because of another player's compromising in this bargaining.

Similarly, the coefficient of the uncooperative emotions $\beta$, represent the degree of influence effecting on the players by the uncooperative emotions. For the player $i$ in round $t$ of bargaining, when $\delta_{i i}(t-1)<\delta_{j i}(t-1)$, player $j$ is more confident than $i$, so the player $i$ will feel angry, desperate, or just scared because of another player's threatening in this bargaining.

Necessary to explain here that, in round $t$ of bargaining, player $i$ will get his own confidence degree according to both confidence degree of the two players' in round $t-1$, but player $j$ will get his own confidence degree according to $i$ 's confidence degree in round $t$ and his own confidence degree in round $t-1$. So we can calculate the bargaining confidence degree with the formula as followed:

$$
\begin{gathered}
\delta_{i i}(t)=\delta_{i i}(t-1)-\alpha_{i} \times \max \left\{\delta_{i i}(t-1)-\delta_{j i}(t-1), 0\right\}+\beta_{i} \times \max \left\{\delta_{j i}(t-1)-\delta_{i i}(t-1), 0\right\} \\
\delta_{j j}(t)=\delta_{j j}(t-1)-\alpha_{j} \times \max \left\{\delta_{j j}(t-1)-\delta_{i j}(t), 0\right\}+\beta_{j} \times \max \left\{\delta_{i j}(t)-\delta_{i i}(t-1), 0\right\}
\end{gathered}
$$

and $t \geq 1$.

Especially, when $\delta_{i i}(t)>1\left(\operatorname{or} \delta_{j j}(t)>1\right)$, we will make the $\delta_{i i}(t)=1\left(\operatorname{or} \delta_{j j}(t)=1\right)$. And when $\delta_{i i}(t)<0\left(\operatorname{or} \delta_{j j}(t)<0\right)$, we will make the $\delta_{i i}(t)=0\left(\operatorname{or} \delta_{j j}(t)=0\right)$.

The improvement of the Rubinstein bargaining model with bargaining confidence degree. With the analysis and the calculation of the bargaining confidence degree, the Rubinstein bargaining model can be expressed as followed:

In round $t$ of bargaining, player $i$ 's bargaining price should be more than $\frac{1-\delta_{j i}}{1-\delta_{i i} \delta_{j i}} \pi$, and playerj's bargaining price should be more than $\frac{1-\delta_{i j}}{1-\delta_{j j} \delta_{i j}} \pi$. And the difference between these two bargaining prices will be the main factors which cause the change of the bargaining confidence degree. When the bargaining prices change, the players will make the decision of accepting the agreement, keeping bargaining or giving up the negotiation.

\section{Summary}

In this paper, we focus on the definition and calculation of the bargaining confidence degree based on the limitations of the Rubinstein bargaining model in the relocation disputes. With the improvement on the information asymmetry and dynamic issue, we can narrow the difference between the calculated bargaining price and the one given by the player in reality, and guide the players to give the more reasonable price as well. Besides, the formula of the bargaining confidence degree shows that the players are effected by the subjective factors which makes the bargaining prices are more reasonable and accurate in the comparison with the prices in reality. Besides the improvement mentioned above, we can see that the calculation of the bargaining price is influenced by more factors and the method to calculating the influence of the subjective factors is more complicated and full of randomness and particularity. So these shortages will make the next step of this research.

\section{References}

[1] X. B. Peng, R. Tan, Y. Hu, Analysis of game theory and Countermeasures of urban demolition dispute, J. Journal of Chongqing University (Social Science Edition), 11 (2005) 19-22.

[2] Z. S. Li, L. Weng, on the social and economic interests in the urban house demolition, J. Journal of Anhui University (Philosophy And Social Sciences Edition), 28 (2004) 95-100. 
[3] W. Zhong, In the process of urbanization analysis of the conflict between the interests of the game and to investigate the behavior of land expropriation public governance ideas, J. Modern Economy, 4 (2013) 28-31.

[4] N. John, The Bargaining Problem, J. Econometrica, 1 (1950) 155-162.

[5] A. Rubinstein, Perfect Equilibrium in a Bargaining Model, J. Econometrica, 1 (1982) 97-109.

[6] S. J. Ji, C. J. Zhang, Y. Q. Liang, B. H. Liu, Rational analysis and verification of bilateral bargaining concessions in disposable participant motive, J. Chinese Journal of management science, 21 (2013) 494-501.

[7] R. A. Lambert, C. Leuz, R. E. Verrecchia, Information asymmetry, information precision, and the cost of capital, J. Review of Finance, 16 (2012) 1-29.

[8] S. Lauermann, Dynamic matching and bargaining games: A general approach, J. The American Economic Review, 103 (2013) 663-689. 